\title{
Stratification Analysis and Case-control Study of Relationships between Interleukin-6 Gene Polymorphisms and Cervical Cancer Risk in a Chinese Population
}

\author{
Wen-Jing Shi ${ }^{1}$, Hao Liu ${ }^{2}$, Dan Wu ${ }^{3}$, Zhen-Hua Tang ${ }^{1 *}$, Yu-Chen Shen ${ }^{1}$, Lin Guo ${ }^{4}$
}

\begin{abstract}
Interleukin-6 (IL-6), a central proinflammatory cytokine, maintains immune homeostasis and also plays important roles in cervical cancer. Therefore, we aimed to evaluate any associations of IL-6 gene polymorphisms at positions -174 and $\mathbf{- 5 7 2}$ with predisposition to cervical cancer in a Chinese population. The present hospitalbased case-control study comprised 518 patients with cervical cancer and 518 healthy controls. Polymorphisms of the IL-6 gene were genotyped by polymerase chain reaction-restriction fragment length polymorphism (PCR-RFLP). Patients with cervical cancer had a significantly higher frequency of the IL-6 -174 CC genotype [odds ratio $(\mathrm{OR})=1.52,95 \%$ confidence interval $(\mathrm{CI})=1.06-2.19 ; p=0.02]$, IL-6 $-572 \mathrm{CC}$ genotype $(\mathrm{OR}=1.91$, 95\% CI $=1.16-3.13 ; p=0.01)$ and IL-6 $-174 \mathrm{C}$ allele $(\mathrm{OR}=1.21,95 \% \mathrm{CI}=1.02-1.44 ; p=0.03)$ compared to healthy controls. When stratifying by the FIGO stage, patients with III-IV cervical cancer had a significantly higher frequency of IL-6 -174 CC genotype $(\mathrm{OR}=1.64,95 \% \mathrm{CI}=1.04-2.61 ; p=0.04)$. The $\mathrm{CC}$ genotypes of the IL-6 gene polymorphisms at positions $\mathbf{- 1 7 4}$ and $\mathbf{- 5 7 2}$ may confer a high risk of cervical cancer. Additional studies with detailed human papillomavirus (HPV) infection data are warranted to validate our findings.
\end{abstract}

Keywords: Interleukin-6 - cervical cancer - gene polymorphism - risk - Chinese females

Asian Pac J Cancer Prev, 15 (17), 7357-7362

\section{Introduction}

Cervical cancer was the third most common cancer on both incidence and mortality for females, accounting for $9 \%(529,800)$ of the total new cancer cases and $8 \%(275$, 100) of the total cancer deaths among females (Jemal et al., 2011). Epidemiological observations had established an aetiological association between human papillomavirus (HPV) infection and cervical cancer (Liu et al., 2011). However, only a small percentage of infected women would finally develop into cervical cancer (Barbisan et al., 2012). Therefore, a hypothesis can be made here that genetic factors and host immune response are also involved in the progression of cervical precancerous lesions to invasive cervical cancer (Yang et al., 2012; Zhang et al., 2013).

Interleukin-6 (IL-6), an important pro-inflammatory cytokine, is a multifunctional protein principally involved in the genesis and maintenance of the inflammatory response (Hamaguchi et al., 2011; Ma et al., 2011; Mosaad et al., 2012; Tao et al., 2012; Crosbie et al., 2013). The human IL-6 gene is organized into five exons and four introns and maps to the short arm of chromosome 7 (7p21) (Bowcock et al., 1988). The IL-6 gene presents two biallelic polymorphisms at positions -174 and -572 in its promoter region (Huang et al., 1999; Bennermo et al., 2004). It had been found that IL-6 promoter polymorphisms (-174G/C and $-572 \mathrm{G} / \mathrm{C})$ were associated with various risks of cancers (Xu et al., 2011; Magalhaes et al., 2013). It was recently suggested that the IL-6 gene polymorphisms at positions -174 and -572 might increase the risk of gynecological malignancies, including cervical cancer (Nogueira de Souza et al., 2006; Castro et al., 2009; Gangwar et al., 2009; Shi et al., 2013). Therefore, we aimed to evaluate the association of the IL-6 gene polymorphisms at positions -174 and -572 with predisposition to cervical cancer in a Chinese population.

\section{Materials and Methods}

\section{Study population}

The present hospital-based case-control study comprised 518 patients with cervical cancer and 518 healthy controls between February 2011 and October 2013 in the International Peace Maternity and Child Health Hospital of China Welfare Institute (IPMCH) and

${ }^{1}$ Department of Clinical Laboratory, ${ }^{3}$ Department of Obstetrics and Gynecology, International Peace Maternity and Child Health Hospital of China Welfare Institute, Shanghai Jiaotong University School of Medicine, ${ }^{4}$ Department of Clinical Laboratory, Fudan University Shanghai Cancer Center, Shanghai Medical College of Fudan University, Shanghai, ${ }^{2}$ Department of Health Technology and Informatics, The Hong Kong Polytechnic University, Hong Kong Kowloon, China *For correspondence: 13774261531@163.com 
Fudan University Shanghai Cancer Center (FUSCC). Healthy female controls were recruited when they were attending a clinic for routine examination. All cases were histopathologically confirmed as primary cervical cancer independently by two gynecologic pathologists. For the cases, clinical and pathological information was extracted including tumor histology, International Federation of Gynecology and Obstetrics (FIGO) stage, tumor size (the largest tumor diameter of the primary tumor), pelvic lymph node metastasis, lympho-vascular space invasion, depth of cervical stromal invasion and the expression of estrogen receptor and progesterone receptor. All individuals were interviewed by trained nurse-interviewers using a structured questionnaire that detailed their age, body mass index (BMI) and menopausal status. The Ethical Committee of the IPMCH approved the study protocols. And all participants also provided written informed consents according to the Declaration of Helsinki.

\section{DNA extraction and genotyping}

The commercially available Qiagen kit (QIAGEN Inc., Valencia, CA, USA) was used to extract DNA from peripheral blood leukocytes. A single base pair (bp) polymorphism at -174 and -572 in the promoter region of the IL- 6 gene was analyzed by the polymerase chain reaction-restriction fragment length polymorphism (PCR-RFLP). The polymorphic region containing the NlaIII restriction site at position -174 base pairs from the

Table 1. Distribution of Characteristics of Cervical Cancer Cases and Healthy Controls

\begin{tabular}{|c|c|c|c|}
\hline Parameters & Controls & Cervical cancer cases & $p$ value \\
\hline Number of subjects & 518 & 518 & \\
\hline Age (years) & $54.9 \pm 9.8$ & $55.2 \pm 10.1$ & 0.63 \\
\hline \multicolumn{4}{|l|}{$\mathrm{BMI}, \mathrm{kg} / \mathrm{m} 2(\%)$} \\
\hline$<25$ & $397(76.6)$ & $406(78.4)$ & 0.5 \\
\hline$\geq 25$ & $121(23.4)$ & $112(21.6)$ & \\
\hline \multicolumn{4}{|c|}{ Menopausal status (\%) } \\
\hline Premenopausal & $371(71.6)$ & $380(73.4)$ & 0.53 \\
\hline Postmenopausal & $147(28.4)$ & $138(26.6)$ & \\
\hline \multicolumn{4}{|l|}{ Histology (\%) } \\
\hline CINIII & & $54(10.4)$ & \\
\hline Squamous & & $418(80.7)$ & \\
\hline Non-squamous & & $46(8.9)$ & \\
\hline \multicolumn{4}{|l|}{ FIGO stage (\%) } \\
\hline I & & $247(47.7)$ & \\
\hline II & & $181(34.9)$ & \\
\hline III-IV & & $90(17.4)$ & \\
\hline \multicolumn{4}{|l|}{ Tumor size, $\mathrm{cm}(\%)$} \\
\hline$<4$ & & $317(61.2)$ & \\
\hline$\geq 4$ & & $201(38.8)$ & \\
\hline \multicolumn{4}{|c|}{ Pelvic lymph node $(\%)$} \\
\hline Negative & & $384(74.1)$ & \\
\hline Positive & & $134(25.9)$ & \\
\hline \multicolumn{4}{|c|}{ Lympho-vascular space invasion (\%) } \\
\hline Negative & & $298(57.5)$ & \\
\hline Positive & & $220(42.5)$ & \\
\hline \multicolumn{4}{|c|}{ Depth of cervical stromal invasion $(\%)$} \\
\hline Negative & & $230(44.4)$ & \\
\hline Positive & & $288(55.6)$ & \\
\hline \multicolumn{4}{|c|}{ Estrogen receptor expression (\%) } \\
\hline Negative & & $351(67.8)$ & \\
\hline Positive & & $167(32.2)$ & \\
\hline \multicolumn{4}{|c|}{ Progesterone receptor expression (\%) } \\
\hline Negative & & $364(70.3)$ & \\
\hline Positive & & $154(29.7)$ & \\
\hline
\end{tabular}

*Abbreviations: $\mathrm{BMI}=$ body mass index; $\mathrm{FIGO}=$ International Federation of Gynecology and Obstetrics, CIN= cervical intraepithelial neoplasia transcription start site (a 198-bp PCR fragment of the IL-6 promoter region) was amplified as previously described (Fishman et al., 1998). The polymorphic region containing the BsrBI restriction site at position -572 base pairs from the transcription start site was amplified using the following primers: 5'-AGATTCCAAGGGTCACTTG-3' and 5'-AGAAGCAGAACCACTCTTC-3' (a 519-bp PCR fragment of the IL-6 promoter region). A 5\% random sample was reciprocally tested by different persons and the reproducibility was $100 \%$.'

\section{Statistical analysis}

The allele and genotype frequencies of IL-6 gene in patients were compared to healthy controls' by Chisquared test. The Hardy-Weinberg equilibrium was tested for goodness-of-fit of chi-square test with one degree of freedom to compare the observed genotype frequencies among the subjects with the expected genotype frequencies. The Statistical Analysis System software (Version 8; SAS Institute Inc., Cary, NC, USA) was used to perform all of the statistical analysis. Comparisons between groups were made with $\chi^{2}$ test (nominal data) or Student t-test (interval data). The $p$ value less than 0.05 was considered statistically significant.

\section{Results}

\section{Characteristics of participants}

Characteristics of cervical cancer cases and healthy controls were showed in Table 1. No significant differences were found between the cervical cancer cases and healthy controls on age, body mass index (BMI), and menopausal status (Table 1). Other information about cervical cancer patients was also provided.

\section{IL-6 -174 G/C polymorphisms and cervical cancer}

Patients with cervical cancer had a significantly higher frequency of IL-6 -174 CC genotype $(\mathrm{OR}=1.52,95 \% \mathrm{CI}$ $=1.06-2.19 ; p=0.02)$ and IL-6 $-174 \mathrm{C}$ allele $(\mathrm{OR}=1.21$, $95 \% \mathrm{CI}=1.02-1.44 ; p=0.03$ ) than healthy controls (Table 2). When stratifying by the FIGO stage, patients with IIIIV cervical cancer had a significantly higher frequency of IL-6 -174 CC genotype $(\mathrm{OR}=1.64,95 \% \mathrm{CI}=1.04-2.61$; $P=0.04$ ) (Table 3).

Table 2. Genotype and Allele Frequencies of IL-6 Gene Polymorphisms Among Cervical Cancer Cases and Healthy Controls

\begin{tabular}{lrrrr}
\hline Genotypes & Cases $(\%)$ & Controls $(\%)$ & OR (95\%CI) & $p$ value \\
\hline-174 GG & $160(30.9)$ & $181(34.9)$ & 1.00 (Reference) & \\
-174 GC & $253(48.8)$ & $259(50.0)$ & $1.11(0.84-1.45)$ & 0.48 \\
-174 CC & $105(20.3)$ & $78(15.1)$ & $1.52(1.06-2.19)$ & 0.02 \\
-174 G allele & $573(55.3)$ & $621(59.9)$ & 1.00 (Reference) & \\
frequency & & & & \\
-174 C allele & $463(44.7)$ & $415(40.1)$ & $1.21(1.02-1.44)$ & 0.03 \\
frequency & & & & \\
-572 GG & $301(58.1)$ & $310(59.8)$ & 1.00 (Reference) & \\
-572 GC & $167(32.2)$ & $181(35.0)$ & $0.95(0.73-1.24)$ & 0.7 \\
-572 CC & $50(9.7)$ & $27(5.2)$ & $1.91(1.16-3.13)$ & 0.01 \\
-572 G allele & $769(74.2)$ & $801(77.3)$ & $1.00($ Reference) & \\
frequency & & & & \\
-572 C allele & $267(25.8)$ & $235(22.7)$ & $1.18(0.97-1.45)$ & 0.1 \\
frequency & & & & \\
\hline
\end{tabular}


Table 3. Stratification Analysis of IL-6 -174G/C Polymorphisms in Cervical Cancer Cases

\begin{tabular}{|c|c|c|c|c|c|c|c|c|c|c|}
\hline \multirow[t]{2}{*}{ Parameters } & \multirow[t]{2}{*}{ Caese } & \multicolumn{3}{|c|}{ GG } & \multicolumn{3}{|c|}{ GC } & \multicolumn{3}{|c|}{$\mathrm{CC}$} \\
\hline & & $\mathrm{n}(\%)$ & OR $(95 \% \mathrm{CI})$ & $p$ value & $\mathrm{n}(\%)$ & OR $(95 \% \mathrm{CI})$ & $p$ value & $\mathrm{n}(\%)$ & OR $(95 \% \mathrm{CI})$ & $p$ value \\
\hline Menopausal status & 518 & $160(30.9)$ & 1 (Reference) & & $253(48.8)$ & 1 (Reference) & & $105(20.3)$ & 1 (Reference) & \\
\hline Premenopausal & 380 & $121(31.8)$ & $1.03(0.79-1.35)$ & 0.83 & $179(47.1)$ & $0.96(0.76-1.22)$ & 0.76 & $80(21.1)$ & $1.04(0.75-1.43)$ & 0.82 \\
\hline Postmenopausal & 138 & $39(28.3)$ & $0.92(0.62-1.36)$ & 0.66 & $74(53.6)$ & $1.10(0.80-1.51)$ & 0.57 & $25(18.1)$ & $0.89(0.56-1.44)$ & 0.64 \\
\hline Histology & 518 & $160(30.9)$ & 1 (Reference) & & $253(48.8)$ & 1 (Reference) & & $105(20.3)$ & 1 (Reference) & \\
\hline CINIII & 54 & $14(25.9)$ & $0.84(0.45-1.55)$ & 0.58 & $29(53.7)$ & $1.10(0.68-1.77)$ & 0.7 & $11(20.4)$ & $1.01(0.51-1.99)$ & 0.99 \\
\hline Squamous & 418 & $131(31.3)$ & $1.02(0.78-1.32)$ & 0.91 & $201(48.1)$ & $0.99(0.79-1.23)$ & 0.89 & $86(20.6)$ & $1.02(0.74-1.39)$ & 0.93 \\
\hline Non-squamous & 46 & $15(32.6)$ & $1.06(0.57-1.94)$ & 0.86 & $23(50.0)$ & $1.02(0.61-1.73)$ & 0.93 & $8(17.4)$ & $0.86(0.39-1.87)$ & 0.7 \\
\hline FIGO stage & 518 & $160(30.9)$ & 1 (Reference) & & $253(48.8)$ & 1 (Reference) & & $105(20.3)$ & 1 (Reference) & \\
\hline I & 247 & $79(32.0)$ & $1.04(0.76-1.41)$ & 0.83 & $126(51.0)$ & $1.04(0.80-1.36)$ & 0.75 & $42(17.0)$ & $0.84(0.57-1.24)$ & 0.38 \\
\hline II & 181 & $56(31.0)$ & $1.00(0.71-1.42)$ & 0.99 & $92(50.8)$ & $1.04(0.78-1.39)$ & 0.79 & $33(18.2)$ & $0.90(0.59-1.38)$ & 0.63 \\
\hline III-IV & 90 & $25(27.8)$ & $0.90(0.56-1.45)$ & 0.66 & 35 (38.9) & $0.80(0.52-1.21)$ & 0.29 & $30(33.3)$ & $1.64(1.04-2.61)$ & 0.04 \\
\hline Tumor size, $\mathrm{cm}$ & 518 & $160(30.9)$ & 1 (Reference) & & $253(48.8)$ & 1 (Reference) & & $105(20.3)$ & 1 (Reference) & \\
\hline$<4$ & 317 & $101(31.9)$ & $1.03(0.78-1.37)$ & 0.83 & $155(48.9)$ & $1.00(0.78-1.28)$ & 0.99 & $61(19.2)$ & $0.79(0.55-1.14)$ & 0.21 \\
\hline$\geq 4$ & 201 & $59(29.4)$ & $0.95(0.68-1.34)$ & 0.77 & $98(48.7)$ & $1.00(0.75-1.33)$ & 0.99 & $44(21.9)$ & $1.08(0.73-1.59)$ & 0.7 \\
\hline Pelvic lymph node & 518 & $160(30.9)$ & 1 (Reference) & & $253(48.8)$ & 1 (Reference) & & $105(20.3)$ & 1 (Reference) & \\
\hline Negative & 384 & $117(30.5)$ & $0.99(0.75-1.30)$ & 0.92 & $191(49.7)$ & $1.02(0.81-1.28)$ & 0.88 & $76(19.8)$ & $0.98(0.71-1.35)$ & 0.89 \\
\hline Positive & 134 & $43(32.1)$ & $1.04(0.71-1.53)$ & 0.85 & $62(46.3)$ & $0.95(0.68-1.33)$ & 0.75 & $29(21.6)$ & $1.07(0.68-1.68)$ & 0.78 \\
\hline $\begin{array}{l}\text { Lympho-vascular } \\
\text { space invasion }\end{array}$ & 518 & $160(30.9)$ & 1 (Reference) & & $253(48.8)$ & 1 (Reference) & & $105(20.3)$ & 1 (Reference) & \\
\hline Negative & 298 & $90(30.2)$ & $0.98(0.73-1.31)$ & 0.88 & $143(48.0)$ & $0.98(0.77-1.26)$ & 0.89 & $65(21.8)$ & $1.08(0.77-1.51)$ & 0.67 \\
\hline Positive & 220 & $70(31.8)$ & $1.03(0.75-1.42)$ & 0.86 & $110(50.0)$ & $1.02(0.78-1.35)$ & 0.87 & $40(18.2)$ & $0.90(0.60-1.33)$ & 0.59 \\
\hline $\begin{array}{l}\text { Depth of cervical } \\
\text { stromal invasion }\end{array}$ & 518 & $160(30.9)$ & 1 (Reference) & & $253(48.8)$ & 1 (Reference) & & $105(20.3)$ & 1 (Reference) & \\
\hline$\leq 1 / 2$ & 230 & $70(30.4)$ & $0.99(0.72-1.36)$ & 0.93 & $115(50.0)$ & $1.02(0.78-1.34)$ & 0.87 & 45 (19.6) & $0.97(0.66-1.42)$ & 0.86 \\
\hline$>1 / 2$ & 288 & $90(31.3)$ & $1.01(0.75-1.36)$ & 0.94 & $138(47.9)$ & $0.98(0.76-1.26)$ & 0.88 & $60(20.8)$ & $1.03(0.73-1.46)$ & 0.88 \\
\hline $\begin{array}{l}\text { Estrogen receptor } \\
\text { expression }\end{array}$ & 518 & $160(30.9)$ & 1 (Reference) & & $253(48.8)$ & 1 (Reference) & & $105(20.3)$ & 1 (Reference) & \\
\hline Negative & 351 & $107(30.5)$ & $0.99(0.75-1.31)$ & 0.93 & $176(50.1)$ & $1.03(0.81-1.30)$ & 0.83 & $68(19.4)$ & $0.96(0.68-1.34)$ & 0.79 \\
\hline Positive & 167 & $53(31.7)$ & $1.03(0.72-1.47)$ & 0.88 & $77(46.1)$ & $0.94(0.69-1.29)$ & 0.72 & $37(22.2)$ & $1.09(0.72-1.65)$ & 0.67 \\
\hline $\begin{array}{l}\text { Progesterone } \\
\text { receptor expression }\end{array}$ & 518 & $160(30.9)$ & 1 (Reference) & & $253(48.8)$ & 1 (Reference) & & $105(20.3)$ & 1 (Reference) & \\
\hline Negative & 364 & $111(30.5)$ & $0.99(0.75-1.30)$ & 0.93 & $176(48.4)$ & $0.99(0.78-1.25)$ & 0.93 & $77(21.1)$ & $1.04(0.76-1.44)$ & 0.8 \\
\hline Positive & 154 & $49(31.8)$ & $1.03(0.71-1.49)$ & 0.87 & $77(50.0)$ & $1.34(0.80-2.26)$ & 0.27 & $28(18.2)$ & $0.90(0.57-1.41)$ & 0.64 \\
\hline
\end{tabular}

*Abbreviations: FIGO= International Federation of Gynecology and Obstetrics; CIN= cervical intraepithelial neoplasia.

Table 4. Stratification Analysis of IL-6-572G/C Polymorphisms in Cervical Cancer Cases

\begin{tabular}{|c|c|c|c|c|c|c|c|c|c|c|}
\hline \multirow[t]{2}{*}{ Parameters } & \multirow[t]{2}{*}{ Cases } & \multicolumn{3}{|c|}{ GG } & \multicolumn{3}{|c|}{ GC } & \multicolumn{3}{|c|}{$\mathrm{CC}$} \\
\hline & & $\mathrm{n}(\%)$ & OR $(95 \% \mathrm{CI})$ & $p$ value & $\overline{\mathrm{n}(\%)}$ & OR $(95 \% \mathrm{CI})$ & $\overline{p \text { value }}$ & $\mathrm{n}(\%)$ & OR $(95 \% \mathrm{CI})$ & $p$ value \\
\hline Menopausal status & 518 & $301(58.1)$ & 1 (Reference) & & $167(32.2)$ & 1 (Reference) & & $50(9.7)$ & 1 (Reference) & \\
\hline Premenopausal & 380 & $218(57.4)$ & $0.99(0.79-1.23)$ & 0.91 & $127(33.4)$ & $1.04(0.80-1.35)$ & 0.79 & $35(9.2)$ & $0.95(0.61-1.50)$ & 0.84 \\
\hline Postmenopausal & 138 & $83(60.1)$ & $1.04(0.76-1.41)$ & 0.83 & $40(29.0)$ & $0.90(0.61-1.33)$ & 0.6 & $15(10.9)$ & $1.13(0.61-2.07)$ & 0.7 \\
\hline Histology & 518 & $301(58.1)$ & 1 (Reference) & & $167(32.2)$ & 1 (Reference) & & $50(9.7)$ & 1 (Reference) & \\
\hline CINIII & 54 & $30(55.6)$ & $0.96(0.60-1.53)$ & 0.85 & $18(33.3)$ & $1.03(0.59-1.81)$ & 0.91 & $6(11.1)$ & $1.15(0.47-2.81)$ & 0.76 \\
\hline Squamous & 418 & $246(58.9)$ & $1.01(0.82-1.25)$ & 0.91 & $133(31.8)$ & $0.99(0.76-1.28)$ & 0.92 & $39(9.3)$ & $0.97(0.62-1.50)$ & 0.88 \\
\hline Non-squamous & 46 & $25(54.3)$ & $0.94(0.56-1.55)$ & 0.8 & $16(34.8)$ & $1.08(0.60-1.96)$ & 0.8 & $5(10.9)$ & $1.13(0.43-2.96)$ & 0.81 \\
\hline FIGO stage & 518 & $301(58.1)$ & 1 (Reference) & & $167(32.2)$ & 1 (Reference) & & $50(9.7)$ & 1 (Reference) & \\
\hline I & 247 & $141(57.1)$ & $0.98(0.76-1.26)$ & 0.89 & $83(33.6)$ & $1.04(0.77-1.41)$ & 0.79 & $23(9.3)$ & $0.97(0.58-1.62)$ & 0.89 \\
\hline II & 181 & $109(60.2)$ & $1.04(0.79-1.37)$ & 0.8 & $55(30.4)$ & $0.94(0.67-1.34)$ & 0.74 & $17(9.4)$ & $0.97(0.55-1.73)$ & 0.93 \\
\hline III-IV & 90 & $51(56.7)$ & $0.98(0.67-1.41)$ & 0.9 & $29(32.2)$ & $1.00(0.64-1.57)$ & 1 & $10(11.1)$ & $1.15(0.56-2.35)$ & 0.7 \\
\hline Tumor size, $\mathrm{cm}$ & 518 & $301(58.1)$ & 1 (Reference) & & $167(32.2)$ & 1 (Reference) & & $50(9.7)$ & 1 (Reference) & \\
\hline$<4$ & 317 & $182(57.4)$ & $0.99(0.78-1.25)$ & 0.92 & $107(33.8)$ & $1.05(0.79-1.39)$ & 0.75 & $28(8.8)$ & $0.92(0.56-1.48)$ & 0.72 \\
\hline$\geq 4$ & 201 & $119(59.2)$ & $1.02(0.78-1.33)$ & 0.89 & $60(29.9)$ & $0.93(0.66-1.30)$ & 0.65 & $22(10.9)$ & $1.13(0.67-1.92)$ & 0.64 \\
\hline Pelvic lymph node & 518 & $301(58.1)$ & 1 (Reference) & & $167(32.2)$ & 1 (Reference) & & $50(9.7)$ & 1 (Reference) & \\
\hline Negative & 384 & $222(57.8)$ & $1.00(0.80-1.24)$ & 0.96 & $126(32.8)$ & $1.02(0.78-1.33)$ & 0.9 & $36(9.4)$ & $0.97(0.62-1.52)$ & 0.9 \\
\hline Positive & 134 & $79(59.0)$ & $1.02(0.74-1.39)$ & 0.93 & $41(30.6)$ & $0.95(0.64-1.40)$ & 0.79 & $14(10.4)$ & $1.08(0.58-2.02)$ & 0.8 \\
\hline $\begin{array}{l}\text { Lympho-vascular } \\
\text { space invasion }\end{array}$ & 518 & $301(58.1)$ & 1 (Reference) & & $167(32.2)$ & 1 (Reference) & & $50(9.7)$ & 1 (Reference) & \\
\hline Negative & 298 & $167(56.1)$ & $0.96(0.76-1.22)$ & 0.76 & $99(33.2)$ & $1.03(0.77-1.37)$ & 0.84 & $32(10.7)$ & $1.11(0.70-1.77)$ & 0.65 \\
\hline Positive & 220 & $134(60.9)$ & $1.05(0.81-1.36)$ & 0.72 & $68(30.9)$ & $0.96(0.69-1.32)$ & 0.8 & $18(8.2)$ & $0.85(0.48-1.49)$ & 0.56 \\
\hline $\begin{array}{l}\text { Depth of cervical } \\
\text { stromal invasion }\end{array}$ & 518 & $301(58.1)$ & 1 (Reference) & & $167(32.2)$ & 1 (Reference) & & $50(9.7)$ & 1 (Reference) & \\
\hline$\leq 1 / 2$ & 230 & $133(57.8)$ & $1.00(0.77-1.29)$ & 0.97 & $78(33.9)$ & $1.05(0.77-1.44)$ & 0.75 & $19(8.3)$ & $0.86(0.49-1.48)$ & 0.58 \\
\hline$>1 / 2$ & 288 & $168(58.3)$ & $1.00(0.79-1.27)$ & 0.98 & $89(30.9)$ & $0.96(0.71-1.29)$ & 0.78 & $31(10.8)$ & $1.12(0.70-1.79)$ & 0.65 \\
\hline $\begin{array}{l}\text { Estrogen receptor } \\
\text { expression }\end{array}$ & 518 & $301(58.1)$ & 1 (Reference) & & $167(32.2)$ & 1 (Reference) & & $50(9.7)$ & 1 (Reference) & \\
\hline Negative & 351 & $198(56.4)$ & $0.97(0.78-1.22)$ & 0.8 & $117(33.3)$ & $1.03(0.79-1.36)$ & 0.81 & $36(10.3)$ & $1.06(0.68-1.67)$ & 0.79 \\
\hline Positive & 167 & $103(61.7)$ & $1.06(0.80-1.41)$ & 0.68 & $50(29.9)$ & $0.93(0.65-1.33)$ & 0.69 & $14(8.4)$ & $0.87(0.47-1.61)$ & 0.66 \\
\hline $\begin{array}{l}\text { Progesterone receptor } \\
\text { expression }\end{array}$ & 518 & $301(58.1)$ & 1 (Reference) & & $167(32.2)$ & 1 (Reference) & & $50(9.7)$ & 1 (Reference) & \\
\hline Negative & 364 & $206(56.6)$ & $0.97(0.78-1.22)$ & 0.82 & $120(33.0)$ & $1.02(0.78-1.34)$ & 0.87 & $38(10.4)$ & $1.08(0.70-1.68)$ & 0.73 \\
\hline Positive & 154 & $95(61.7)$ & $1.06(0.79-1.42)$ & 0.69 & $47(30.5)$ & $0.95(0.65-1.37)$ & 0.77 & $12(7.8)$ & $0.81(0.42-1.55)$ & 0.52 \\
\hline
\end{tabular}

*Abbreviations: FIGO= International Federation of Gynecology and Obstetrics; CIN= cervical intraepithelial neoplasia 
Table 5. Previous Studies on the Association between Cervical Cancer Risk and IL-6 Gene Polymorphisms After Compared with Healthy Controls

\begin{tabular}{|c|c|c|c|c|c|c|c|}
\hline Publish year & Genotype & Ethnicity (n) & Category & Cancer stage & OR $(95 \% \mathrm{CI})$ & $p$ value & References \\
\hline 2006 & IL-6-174G/C & Brazilian $(n=56)$ & $\mathrm{GC}+\mathrm{CC}$ & Total & $1.88(1.1-3.4)$ & 0.035 & $\begin{array}{l}\text { (Nogueira de } \\
\text { Souza et al., 2006) }\end{array}$ \\
\hline 2009 & IL-6-174G/C & Indian $(n=160)$ & $\begin{array}{l}\mathrm{CC} \\
\mathrm{GC}\end{array}$ & $\begin{array}{l}\text { Total } \\
\text { Stage I }\end{array}$ & $\begin{array}{l}3.16(1.26-7.92) \\
3.63(1.54-8.56)\end{array}$ & $\begin{array}{l}0.014 \\
0.003\end{array}$ & (Gangwar et al., 2009) \\
\hline 2011 & IL-6-174G/C & Australian $(\mathrm{n}=131)$ & $\mathrm{G} / \mathrm{C}$ & CIN II+ & $1.0(0.6-1.5)$ & 0.9 & (Grimm et al., 2011) \\
\hline 2013 & IL-6-174G/C & $\begin{array}{c}\text { Chinese (Study 1: } \\
\text { n=458; Study 2: } \\
\text { n=1126) }\end{array}$ & $\begin{array}{l}\mathrm{GC} \\
\mathrm{CC}\end{array}$ & Total & $\begin{array}{l}\text { Study } 1: \\
2.10(0.21-20.76) \\
2.79(0.29-26.93) \\
\text { Study } 2: \\
2.64(0.70-9.94) \\
2.73(0.74-10.11)\end{array}$ & $\begin{array}{l}\text { Study } 1: \\
0.524 \\
0.374 \\
\text { Study 2: } \\
0.151 \\
0.132\end{array}$ & (Shi et al., 2013) \\
\hline
\end{tabular}

\section{IL-6 -572 G/C polymorphisms and cervical cancer}

Patients with cervical cancer had a significantly higher frequency of IL-6 -572 CC genotype $(\mathrm{OR}=1.91$, $95 \% \mathrm{CI}=1.16-3.13 ; p=0.01)$ than healthy controls. When stratifying by the menopausal status, histology, FIGO stage, tumor size, pelvic lymph node, lympho-vascular space invasion, depth of cervical stromal invasion, estrogen receptor expression and progesterone receptor expression of cervical cancer, no obvious statistical association was found (Table 4).

\section{Discussion}

This study provided an evidence for a role of genetic factors in the development of cervical cancer. A metaanalysis of thirteen studies with 1,936 cases and 2, 858 controls indicated that the methylenetetrahydrofolate reductase (MTHFR) $677 \mathrm{~T}$ allele might enhance the risk of cervical cancer in the Asian female population and played a protective role in Caucasian females (Zhu et al., 2013). A meta-analysis of fifteen studies with a total of 3, 743 cervical cancer cases and 4, 096 controls suggested that tumor necrosis factor alpha (TNF-alpha) $308 \mathrm{G} / \mathrm{A}$ polymorphism was associated with increased risk of cervical cancer, and TNF-alpha $308 \mathrm{G} / \mathrm{A}$ mutant allele A was a risk factor of cervical cancer (Zhang et al., 2013). A meta-analysis of eight studies suggested that the genetic polymorphism in IL-10-592C/A was a risk factor for developing cervical cancer, especially for Asians (Ni et al., 2013). A meta-analysis of twentyeight case-control studies suggested that p53 codon 72 polymorphisms might be associated with increased risk of cervical cancer, especially among Indians (Zhou et al., 2012). A meta-analysis of 21 case-control studies provided strong evidence that the GSTM1 genotype was associated with the development of cervical cancer, especially in smokers, and Chinese and Indian populations (Zhang et al., 2012a).A meta-analysis including 13 studies suggested that the Arg194Trp polymorphism of x-ray repair crosscomplementing protein 1 (XRCC1) increased the risk of cervical cancer (Shuai et al., 2012).

IL-6 promoter polymorphisms were also associated with various cancers risks. A systematic review and meta-analysis of 7 studies inferred that IL-6 -174G/C polymorphism was associated with an increased prostate cancer risk (Zhang et al., 2012b). A meta-analysis involving 29, 377 cases and 37, 739 controls believed that IL-6-174G/C polymorphism was associated with bladder cancer risk (Xu et al., 2011). A case-control study suggested that IL-6 -174G/C polymorphism was associated with the risk of squamous cell esophageal cancer (Upadhyay et al., 2008). A study from the Southwestern United States reported that IL-6 -174G/C polymorphism was associated with breast cancer risk (Slattery et al., 2008). A case-control study informed that IL-6 -174G/C polymorphism was strongly associated with oral cancer risk (Vairaktaris et al., 2006). A study surmised that the IL-6 -174G/C polymorphism might serve as an additional prognostic parameter in patients with vulvar cancer (Grimm et al., 2005). A case-control study from Spain assumed that IL-6 -174G/C polymorphism was associated with colorectal cancer risk (Landi et al., 2003).

However, it is still unclear that what is the association or molecular mechanism between IL-6 promoter polymorphisms $(-174 \mathrm{G} / \mathrm{C}$ and $-572 \mathrm{G} / \mathrm{C})$ and the risk of cervical cancer. It is well-known that host immune response and chronic inflammation play critical roles on preventing the progression of cervical cancer (Shi et al., 2013). IL-6, an important pro-inflammatory cytokine, is a multifunctional protein principally involved in the genesis and maintenance of the inflammatory response (Kyo et al., 2000; Srivani et al., 2003; Shi et al., 2013). There is local production of IL-6 in cervicovaginal secretions. And the production of IL-6 was believed to be related to the severity of cervical neoplasia (Tjiong et al., 1999). Substantially high microenvironmental IL-6 levels promoted tumor angiogenesis and the development of cervical cancer (Wei et al., 2001a). IL-6 regulated the mcl-1 expression via a PI 3-K/Akt-dependent pathway that might facilitate the oncogenesis of human cervical cancer by modulating the apoptosis threshold (Wei et al., 2001b). It was reported that IL-6 $-174 \mathrm{G} / \mathrm{C}$ polymorphism could affect the transcription rate of a reporter gene in transient transfection studies, which could be associated with the different IL-6 responses to stressful stimuli (Fishman et al., 1998). In table 5, previous studies about the association between IL-6 -174G/C and cervical cancer risk were summarized, which indicated that ethnicities or region differences might be an important influential factor. On the other hand, relative studies were not systematic with small sample size. The paradox of final results was existed between our study and the study from Shi TY's study (Shi et al., 2013). Considering the relative data of GG genotype as reference only came from 1 case in sub-study 1 and 3 cases in sub-study 2, so the final analysis on GC and CC was not convincing. 
Some limitations of this study should be noted. First of all, these results should be interpreted with caution because the population was only from China, which reduced the possibility of confounding from ethnicity, so it did not permit extrapolation of the results to other ethnic groups. Secondly, it was the lack of routine HPV screening. We did not have enough information on other risk factors, especially HPV infection, which could be potential confounder. At last, this was a two hospitalbased case-control study, so the selection bias was not avoidable and the subjects might not be representative for the general population.

In conclusion, the CC genotype of the IL-6 gene polymorphisms at positions -174 and -572 may confer a high risk of cervical cancer. Additional studies with detailed HPV infection data are warranted to validate our findings.

\section{Acknowledgements}

This research received grant from Natural Science Foundation of China (NSFC, No: 09ZR1435000). The authors declare that they have no conflict of interests.

\section{References}

Barbisan G, Perez LO, Contreras A, Golijow CD (2012). TNF-o and IL-10 promoter polymorphisms, HPV infection, and cervical cancer risk. Tumour Biol, 33, 1549-56.

Bennermo M, Held C, Green F, et al (2004). Prognostic value of plasma interleukin- 6 concentrations and the $-174 \mathrm{G}>\mathrm{C}$ and $-572 \mathrm{G}>\mathrm{C}$ promoter polymorphisms of the interleukin- 6 gene in patients with acute myocardial infarction treated with thrombolysis. Atherosclerosis, 174, 157-63.

Bowcock AM, Kidd JR, Lathrop GM, et al (1988). The human "interferon-beta 2 /hepatocyte stimulating factor/ interleukin-6" gene: DNA polymorphism studies and localization to chromosome 7p21. Genomics, 3, 8-16.

Castro FA, Haimila K, Sareneva I, et al (2009). Association of HLA-DRB1, interleukin-6 and cyclin D1 polymorphisms with cervical cancer in the Swedish population-a candidate gene approach. Int $J$ Cancer, 125, 1851-8.

Crosbie EJ, Einstein MH, Franceschi S, Kitchener HC (2013). Human papillomavirus and cervical cancer. Lancet, 382, 889-99.

Fishman D, Faulds G, Jeffery R, et al (1998). The effect of novel polymorphisms in the interleukin-6 (IL-6) gene on IL-6 transcription and plasma IL-6 levels, and an association with systemic-onset juvenile chronic arthritis. J Clin Invest, 102, 1369-76.

Gangwar R, Mittal B, Mittal RD (2009). Association of interleukin-6 $-174 \mathrm{G}>\mathrm{C}$ promoter polymorphism with risk of cervical cancer. Int J Biol Markers, 24, 11-6.

Grimm C, Six L, Tomovski C, et al (2005). A common interleukin-6 promoter polymorphism in patients with vulvar cancer. J Soc Gynecol Investig, 12, 617-20.

Grimm C, Watrowski R, Baumühlner K, et al (2011). Genetic variations of interleukin- 1 and -6 genes and risk of cervical intraepithelial neoplasia. Gynecol Oncol, 121, 537-41.

Hamaguchi M, Kawahito Y, Ishino H, et al (2011). Mucin from rheumatoid arthritis synovial fluid enhances interleukin-6 production by human peripheral blood mononuclear cells. Hum Immunol, 72, 241-8.

Huang D, Zheng C, Giscombe R, et al (1999). Polymorphisms at -174 and in the 3 ' flanking region of interleukin-6 (IL-6) gene in patients with myasthenia gravis. J Neuroimmunol, 101, 197-200.

Jemal A, Bray F, Center MM, et al (2011). Global cancer statistics. CA Cancer J Clin, 61, 69-90.

Kyo S, Kanaya T, Takakura M, Inoue M (2000). A case of cervical cancer with aggressive tumor growth: possible autocrine growth stimulation by G-CSF and IL-6. Gynecol Oncol, 78, 383-7.

Landi S, Moreno V, Gioia-Patricola L, et al (2003). Association of common polymorphisms in inflammatory genes interleukin (IL)6, IL8, tumor necrosis factor alpha, NFKB1, and peroxisome proliferator-activated receptor gamma with colorectal cancer. Cancer Res, 63, 3560-6.

Liu D, Zhou P, Zhang L, et al (2011). Differential expression of oct4 in hpv-positive and hpv-negative cervical cancer cells is not regulated by DNA methyltransferase 3a. Tumour Biol, 32, 941-50.

Ma Y, Tang RK, Yang X, et al (2011). Lack of an association between interleukin- 6 gene promoter polymorphisms $(-174 \mathrm{G} / \mathrm{C},-572 \mathrm{G} / \mathrm{C})$ and ischemic heart disease and/or ischemic stroke: a meta-analysis. Hum Immunol, 72, 641-51.

Magalhães JF, Cortinhas AJ, Albuquerque CM, et al (2013). Interleukin- 6 gene $-174 \mathrm{G}>\mathrm{C}$ and $-636 \mathrm{G}>\mathrm{C}$ promoter polymorphisms and prostate cancer risk. Mol Biol Rep, 40, 449-55.

Mosaad YM, Fathy H, Fawzy Z, El-Saied MA (2012). Tumor necrosis factor- $\alpha-308 \mathrm{G}>\mathrm{A}$ and interleukin-6 -174 G>C promoter polymorphisms and pemphigus. Hum Immunol, 73, 560-5.

Ni J, Ye Y, Teng F, Wu Q (2013). Interleukin 10 polymorphisms and cervical cancer risk: a meta-analysis. Int J Gynecol Cancer, 23, 126-33.

Nogueira de Souza NC, Brenna SM, Campos F, et al (2006). Interleukin-6 polymorphisms and the risk of cervical cancer. Int J Gynecol Cancer, 16, 1278-82.

Shi TY, Zhu ML, He J, et al (2013). Polymorphisms of the interleukin 6 gene contribute to cervical cancer susceptibility in eastern Chinese women. Hum Genet, 132, 301-12.

Shuai HL, Luo X, Yan RL, Li J, Chen DL (2012). XRCC1 polymorphisms are associated with cervical cancer risk and response to chemotherapy: a systematic review and metaanalysis. Asian Pac J Cancer Prev, 13, 6423-7.

Slattery ML, Curtin K, Sweeney C, Wolff RK, Baumgartner RN, Baumgartner KB, Giuliano AR, Byers T (2008). Modifying effects of IL-6 polymorphisms on body size-associated breast cancer risk. Obesity, 16, 339-47.

Srivani R, Nagarajan B (2003). A prognostic insight on in vivo expression of interleukin-6 in uterine cervical cancer. Int $J$ Gynecol Cancer, 13, 331-9.

Tao QS, Huang HL, Chai Y, et al (2012). Interleukin-6 upregulates the expression of interleukin-15 is associated with MAPKs and PI3-K signaling pathways in the human keratinocyte cell line, HaCaT. Mol Biol Rep, 39, 4201-5.

Tjiong MY, van der Vange N, ten Kate FJ, et al (1999). Increased IL-6 and IL-8 levels in cervicovaginal secretions of patients with cervical cancer. Gynecol Oncol, 73, 285-91.

Upadhyay R, Jain M, Kumar S, Ghoshal UC, Mittal B (2008). Association of interleukin-6 $(-174 \mathrm{G}>\mathrm{C})$ promoter polymorphism with risk of squamous cell esophageal cancer and tumor location: an exploratory study. Clin Immunol, 128, 199-204.

Vairaktaris E, Yiannopoulos A, Vylliotis A, et al (2006). Strong association of interleukin-6 -174 G>C promoter polymorphism with increased risk of oral cancer. Int J Biol Markers, 21, 246-50.

Wei LH, Kuo ML, Chen CA, et al (2001a). Interleukin-6 in 
Wen-Jing Shi et al

cervical cancer: the relationship with vascular endothelial growth factor. Gynecol Oncol, 82, 49-56.

Wei LH, Kuo ML, Chen CA, et al (2001b). The anti-apoptotic role of interleukin-6 in human cervical cancer is mediated by up-regulation of Mcl-1 through a PI 3-K/Akt pathway. Oncogene, 20, 5799-809.

Xu B, Niu XB, Wang ZD, et al (2011). IL-6 -174G>C polymorphism and cancer risk: a meta-analysis involving 29 , 377 cases and 37, 739 controls. Mol Biol Rep, 38, 2589-96.

Yang S, Jia C, Zhu H, Han S (2012). CYP1A1 Ile462Val polymorphism and cervical cancer: evidence from a metaanalysis. Tumour Biol, 33, 2265-72.

Zhang H, Xu Y, Li L, Liu R, Ma B (2012). The interleukin-6 $-174 \mathrm{G} / \mathrm{C}$ polymorphism and prostate cancer risk: a systematic review and meta-analysis. Urol Int, 88, 447-53.

Zhang HL, Zhang YJ (2013). A systemic assessment of the association between tumor necrosis factor alpha $308 \mathrm{G} / \mathrm{A}$ polymorphism and risk of cervical cancer. Tumor Biol, 34, 1659-65.

Zhang ZY, Jin XY, Wu R, et al (2012). Meta-analysis of the association between GSTM1 and GSTT1 gene polymorphisms and cervical cancer. Asian Pac J Cancer Prev, 13, 815-9.

Zhou X, Gu Y,Zhang SL (2012). Association between p53 codon 72 polymorphism and cervical cancer risk among Asians: a HuGE review and meta-analysis. Asian Pac J Cancer Prev, 13, 4909-14.

Zhu J, Wu L, Kohlmeier M, Ye F, Cai W (2013). Association between MTHFR C677T, MTHFR A1298C and MS A2756G polymorphisms and risk of cervical intraepithelial neoplasia II/III and cervical cancer: a meta-analysis. Mol Med Rep, $\mathbf{8}, 919-27$. 